\section{Is bodyweight associated with the general health of dogs under primary veterinary care in the UK}

\section{Elizabeth Ainsworth, Camilla L. Pegram, Dave C. Brodbelt, David B. Church, Dan G. O'Neill}

RVC, Hertfordshire, United Kingdom

\section{OBJECTIVES}

To evaluate associations between adult bodyweight and longevity and the frequency of common disorders in dogs under UK primary veterinary care.

\section{METHODS}

Demographic and diagnostic data were extracted from anonymised clinical records of randomly selected dogs under primary veterinary care during 2016 in the
VetCompass database. Mean adult ( $>18$ months) bodyweight was extracted for each dog. Multivariable logistic regression analysis evaluated bodyweight as a risk factor for the twelve most common disorders.

\section{RESULTS}

Median adult bodyweight of the 20,752 study dogs was $13.59 \mathrm{~kg}$ (IQR: 8.15-24.83). Median age at death was $12 \mathrm{y}$ (IQR: 9.39-14.04). Longevity increased as bodyweight decreased. Dogs $<10 \mathrm{~kg}$ lived longer than dogs $\geq 40 \mathrm{~kg}$ $(12.64 y, 95 \% \mathrm{Cl}: 11.79-12.47$ vs $9.81 y, 95 \% \mathrm{Cl}: 8.92-9.90$ respectively). The prevalence of having at least one disorder was lowest in dogs $<10 \mathrm{~kg}(4,970 / 7,638,65.1 \%)$. Bodyweight was associated with the odds of 7 of the 12 most common disorders. Dogs $\geq 40 \mathrm{~kg}$ had the lowest odds of dental (odds ratio [OR]: $0.22,95 \% \mathrm{Cl}: 0.16-0.29$ $\mathrm{P}<0.001$ ), claw/nail (OR: 0.64, 95\% Cl0.47-0.86, $\mathrm{P}=$ 0.004 ) and anal sac (OR: $0.32,95 \% \mathrm{Cl}: 0.21-0.50, \mathrm{P}<$ 0.001 ) disorders compared to dogs <10 kg. Increasing bodyweight was associated with increasing odds of musculoskeletal disorder up to $40 \mathrm{~kg}(\mathrm{P}<0.001)$.

\section{STATEMENT (CONCLUSIONS)}

Adult bodyweight is associated with differential longevity and disease risk but breed effects rather than bodyweight itself may explain much of these differences. Awareness of these effects can assist veterinarians to prepare owners of specific breeds for likely health and disorder outcomes.

\section{Chewing the fat: prevalence and risk factors for obesity in dogs under primary veterinary care in the UK}

\section{Alice H Ashworth, Camilla Pegram, Dave C Brodbelt, David B Church, Dan G O'Neill}

RVC, Hertfordshire, United Kingdom

\section{OBJECTIVES}

To estimate prevalence and demographic risk factors for overweight/obesity in dogs under primary veterinary care in the UK.

\section{METHODS}

Electronic patient records of dogs within VetCompass ${ }^{\mathrm{TM}}$ during 2016 were retrospectively evaluated using cross-sectional analysis. A sample of 22,333 dogs from a denominator population of 905,544 was analysed to identify dogs recorded with obesity within the patient records during 2016. Multivariable binary logistic regression modelling was used to identify risk factors.

\section{RESULTS}

Obesity was the 6th most prevalent disorder with a prevalence of $7.1 \%(95 \% \mathrm{Cl}=6.7-7.4)$. Risk factors included neutering, age, bodyweight, insurance, head-shape and breed. There was no association between sex and obesity. Neutered dogs had 1.62 times odds $(95 \% \mathrm{Cl}=1.44-$ 1.81) of obesity compared to entire dogs. Increasing age and increasing bodyweight showed increased odds of obesity. Compared to entire males, neutered males had $1.75(95 \% \mathrm{Cl}=1.50-2.05)$, entire females $1.19(95 \% \mathrm{Cl}=$ $1.00-1.41)$ and neutered females $1.76(95 \% \mathrm{Cl}=1.50$ 2.06) times the odds of obesity. Compared to dolichocephalic dogs, brachycephalics had $1.54(95 \% \mathrm{Cl}=$ $1.20-1.98)$, brachycephalic-crosses $2.05(95 \% \mathrm{Cl}=1.24-$ $3.41)$ and mesocephalics $1.68(95 \% \mathrm{Cl}=1.34-2.10)$ times the odds of obesity. Compared to crossbreeds, four breeds were at increased odds of obesity: Pugs had 3.00 $(95 \% \mathrm{Cl}=2.12-4.08)$, Golden Retrievers $2.46(95 \% \mathrm{Cl}=$ 1.72-3.63), Labradors $1.59(95 \% \mathrm{Cl}=1.30-1.95)$ and $\mathrm{CKCS}$ $1.41(95 \% \mathrm{Cl}=1.01-1.96)$. 